\title{
Perforation of Cecal Diverticulum after Appendectomy
}

\author{
Kesici Ugur
}

\begin{abstract}
Solitary cecal diverticulum was first described by Potier in 1912. Although it is a rare condition, however, there is an increased prevalence in Asian populations. The cecal diverticula are usually asymptomatic, however, 10-20\% of the cases become symptomatic due to complications. The most common diverticulum-related complication is diverticulitis. Other complications include perforation, hemorrhage, and rarely intestinal obstruction. To conclude, cecal diverticulum is a rare condition that usually presents itself with complications. It is mostly diagnosed perioperatively in the cases of acute appendicitis, and rarely preoperatively. Cecal diverticulitis is commonly misdiagnosed as acute appendicitis. This is why USG, CT, and MRI are commonly used in the preoperative diagnosis. There are very few cases that are confirmed to have both cecal diverticulitis and acute appendicitis. When the condition is majorly diagnosed perioperatively after an acute appendicitis diagnosis; it should be remembered that in the case of determining cecal diverticulitis during the appendectomy, the best course of action is to simultaneously apply diverticulectomy. In this case report, a 54-year male patient is presented, where a cecal diverticulum perforation developed in the early postoperative phase following the appendectomy. At the time of admission, the patient had the complaints of distension, extensive sensitivity, and rebound. The patient underwent laparotomy and the diverticulectomy was performed with a linear stapler. In this case, the presence of a combination of acute appendicitis and cecal diverticulitis was confirmed through histopathological examination.
\end{abstract}

Key Words: Cecal diverticulum, Acute appendicitis, Apendectomy, Perforation.

\section{INTRODUCTION}

Solitary cecal diverticulum was first described by Potier in 1912.1 It is a rare condition, however, there is an increased prevalence in Asian populations. ${ }^{1}$ In the Western populations, the incidence of cecal diverticulitis is known to be from 0.9 to $5 \% .2$ Cecal diverticulitis makes up $5 \%$ of all colon diverticulitis cases. ${ }^{3}$ Cristaudo et al. indicated that among 632 colon diverticulitis patients, only $2.06 \%$ had developed cecal diverticulitis. 4 A cecal diverticulum is commonly observed among middle-aged males. The mean age is reported to be 44.5

Cecal diverticulum results from pulsion of the cecum in the 6th week of pregnancy. It is thought to have two types: congenital and acquired. The congenital diverticula are the "true" diverticula, in that they contain all layers of the colonic wall. The acquired diverticula are "pseudo" diverticula, in that they do not contain any muscular layer. ${ }^{2}$

The cecal diverticula are usually asymptomatic; however, $10-20 \%$ of the cases become symptomatic due to complications. The most common diverticulum-related complication is diverticulitis. Other complications include perforation, hemorrhage, and rarely intestinal obstruction. ${ }^{3}$ The medical manifestation of cecal diverticulitis is similar

Department of General Surgery, Faculty of Medicine,

University of Beykent, Istanbul, Turkey

Correspondence: Dr. Kesici Ugur, Department of General

Surgery, Faculty of Medicine, University of Beykent, Istanbul, Turkey

E-mail: ugurkesici77@mynet.com

Received: December 22, 2017; Accepted: July 03, 2018 to that of acute appendicitis. Thus, the correct diagnosis is usually made during the operation. ${ }^{5}$ However, several studies indicate that some clinical characteristic findings may assist the diagnostic process. For example, the pain that is caused by cecal diverticulitis is relatively long in duration, and it typically starts and remains in the lower right quadrant. On the other hand, the pain that is caused by acute appendicitis starts from the central abdomen, and subsequently localises in the lower right quadrant. 5,6

Cecal diverticulitis is commonly misdiagnosed as acute appendicitis. ${ }^{6}$ The patient should be examined through ultrasonography (USG), computerised tomography (CT) and magnetic resonance imaging (MRI) for the preoperative diagnosis of the acute abdominal pathologies. ${ }^{3}$ USG is specifically insufficient for diagnosis among the obese patients with intestinal gas. In these conditions, CT is a more reliable option. It is also more beneficial in the determination of the cecal diverticulitis-related complications, the evaluation of the treatment response, and the percutaneous drainage of the potential abscess that may develop as a complication. MRI should be preferred as an alternative to $\mathrm{CT}$, especially among pregnant women. ${ }^{3}$

Appendectomy is the most commonly performed emergency operation for the cases of primary acute appendicitis. Incidentally, cecal diverticulitis can be perioperatively identified. Approximately $90 \%$ of all cecal diverticulitis cases are perioperatively diagnosed following an acute appendectomy diagnosis. However, the identification of a cecal diverticulum can complicate the surgical process. ${ }^{2}$ Thus, there is not a consensus regarding the best treatment option in the case of cecal 
diverticulitis. The literature indicates several different treatment options, ${ }^{1-3,7}$ that range from a conservative treatment to aggressive surgery. ${ }^{3}$ Chou et al. have classified cecal diverticulitis according to the degree of inflammation, and they have determined the best treatment option for every class. 8 Grade 1: inflamed diverticulitis, Grade 2: inflamed cecal mass, Grade 3: localised abscess and fistula, Grade 4: diffuse peritonitis or free perforation. While diverticulectomy is sufficient for Grade 1 and 2 diseases, right hemicolectomy is recommended for Grades 3 and 4.8

Cecal diverticulitis can be surgically treated with a conventional operation or with laparoscopy. Laparoscopy is now the more popular option, as the surgeons have become more experienced and it is less invasive. 9

In this case report, a 54-year male patient is presented, where a cecal diverticulum perforation developed in the early postoperative phase following the appendectomy.

\section{CASE REPORT}

In this case report, a 54-year male patient is presented, who underwent diverticulectomy due to a cecal diverticulum perforation developed in the early postoperative phase following the appendectomy. The patient was admitted to the hospital following an appendectomy operation that was performed in an epicenter, with the complaints of increasing abdominal pain and swelling. The surgery report from the initial healthcare centre indicated a performed appendectomy having a diverticulum in the cecum. It was indicated that the tip of the cecum was edematous, however, the physicians did not think that a diverticulectomy was necessary. At the time of admission, the patient had the complaints of distension, extensive sensitivity, and rebound. The intraabdominal drainage revealed bilious fluid. The examination findings were as follows: body temperature, $38.6^{\circ} \mathrm{C}$; leukocyte count, 14680 ; and CRP (C-reactive protein), $263.2 \mathrm{mg} / \mathrm{L}$. It was decided that the patient would undergo laparoscopy. The laparoscopic

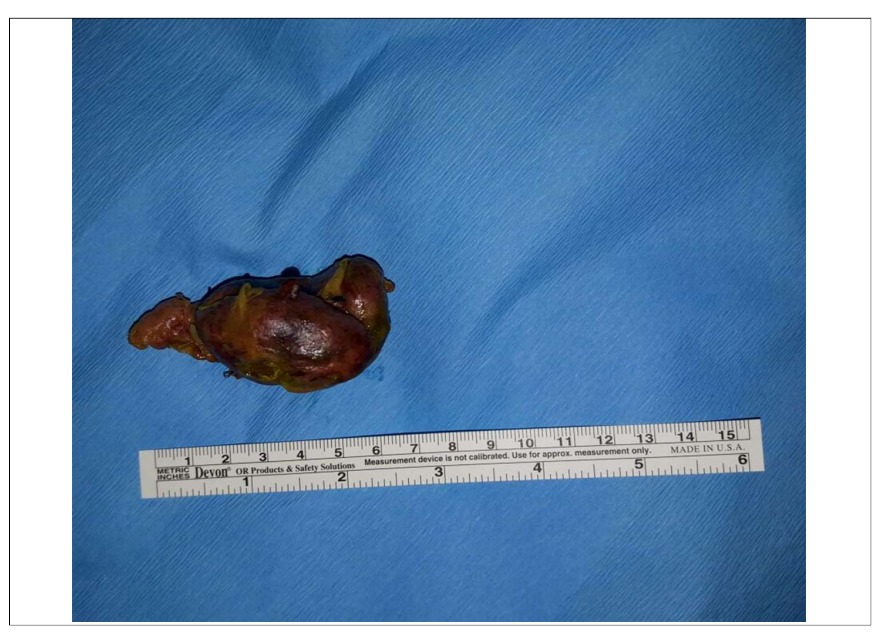

Figure 1: Diverticulectomy specimen. exploration revealed that all the intestinal loops were severely dilated and edematous, and there was extensive bilious fluid in the abdominal cavity. As the operation area was too narrow, it was decided to perform laparotomy. The laparotomy revealed a perforated diverticulum at the cecum, that was approximately 3-4 $\mathrm{cm}$. The colonic wall at the base of the diverticulum was not edematous or inflamed. The diverticulectomy was performed with a linear stapler. The postoperative followup did not indicate any complications other than seroma and fat necrosis. The patient was discharged on the 10th postoperative day. The histopathological evaluation reported a diverticulitis (size: $5 \times 5.5 \mathrm{~cm}$ ). The diverticulitis specimen is presented in Figure 1.

\section{DISCUSSION}

Cecal diverticulitis is a rare condition; however, it should be considered, especially in the differential diagnosis of the right iliac fossa. ${ }^{9}$ Seventy percent of all cecal diverticulitis patients are operated with an acute appendicitis diagnosis. 5,9 Kalcan et al. have retrospectively evaluated 1,163 patients that were operated for acute appendicitis; and found that $6(0.5 \%)$ of these patients had cecal diverticulitis. ${ }^{2}$ It was reported that 5 of these patients' appendicitis had a regular macroscopic appearance, and only 1 patient was diagnosed with suppurative appendicitis. This indicates that there are very rare cases that are histopathologically confirmed to have both cecal diverticulitis and acute appendicitis. In this case, the presence of a combination of acute appendicitis and cecal diverticulitis was confirmed through histopathological examination.

Only $1 \%$ of the cecal diverticulitis cases is preoperatively diagnosed. Most commonly (appx. 90\%), the correct diagnosis is made perioperatively following the acute appendectomy diagnosis. ${ }^{2}$ In this case, cecal diverticulitis was determined during an appendectomy. The literature indicates several different treatment options, $1-3,7$ for the treatment of cecal diverticulitis. Fang et al. recommend a wide resection; because in $29 \%$ of the patients that only undergo an appendectomy, ${ }^{7}$ the rate of recurrent diverticulitis and the rate of a requirement for a right hemicolectomy is $12.5 \%$. In this case, the patient underwent appendectomy following the primary acute appendicitis diagnosis. Cecal diverticulitis was perioperatively identified, but the surgeons did not perform diverticulectomy as the diverticulum was not perforated. However, the diverticulum perforated in the early postoperative stage, and the patient subsequently developed generalised peritonitis, leading to a relaparotomy and diverticulectomy. It is known that this condition increased the risk of morbidity and mortality. In the light of the clinical manifestations and the literature,2,3 diverticulectomy should be considered as an option when cecal diverticulitis is determined during an appendectomy. During the re-laparotomy, the patient 
was determined to have a Grade 4 cecal diverticulitis, according to the Chou et al. classification. ${ }^{8}$ Diverticulectomy was performed as the base of the diverticulum was not inflamed, and there were no postoperative complications.

The initial surgical method was laparoscopic surgery; however, as there were extensive dilatation and intraabdominal intestinal fluids, the surgeons switched to conventional surgery.

To conclude, cecal diverticulum is a rare medical condition which usually presents itself with complications. It should be remembered that if cecal diverticulitis is identified during appendectomy, diverticulectomy can be an adequate treatment option.

\section{REFERENCES}

1. Ruiz-Tovar J, Reguero-Callejas ME, González Palacios F. Inflammation and perforation of a solitary diverticulum of the cecum. A report of 5 cases and literature review. Rev Esp Enferm Dig 2006; 98:875-80.

2. Kalcan S, Basak F, Hasbahçeci M, Kiliç A, Canbak T, Kudas I, et al. Intraoperative diagnosis of cecal diverticulitis during surgery for acute appendicitis: Case series. Ulus Cerrahi Derg 2016; 32:54-7.

3. Hot S, Egin S, Gökçek B, Yesiltas M, Alemdar A, Akan A, et al. Solitary caecum diverticulitis mimicking acute appendicitis. Ulus Travma Acil Cerrahi Derg 2015; 21:520-3.

4. Cristaudo A, Pillay P, Naidu S. Caecal diverticulitis: Presentation and management. Ann Med Surg (Lond) 2015; 4:72-5.

5. Kyziridis DS, Parpoudi SN, Antoniou ND, Konstantaras DCh, Moysidis MG, Christoforidis E Ch, et al. Cecal diverticulitis is a challenging diagnosis: a report of 3 cases. Am J Case Rep 2015; 16:206-10.

6. Cole M, Ayantunde AA, Payne J. Cecal diverticulitis presenting as acute appendicitis: A case report. World J Emerg Surg 2009; 4:29.

7. Fang JF, Chen RJ, Lin BC, Hsu YB, Kao JL, Chen MF. Aggressive resection is indicated for cecal diverticulitis. Am J Surg 2003; 185:135-40.

8. Chou YH, Chiou HJ, Tiu CM, Chen JD, Hsu CC, Lee CH, et al. Sonography of acute right side colonic diverticulitis. Am J Surg 2001; 181:122-7.

9. Kroening HL, Rai S. Caecal diverticulitis, an uncommon mimic of appendicitis. BMJ Case Rep 2013; 6:bcr2012007820. 